Teodor Vasić1, Nikola Topalović, Biljana Đurić, Sanja Mazić

\title{
THE INFLUENCE OF MUSIC ON CARDIOPULMONARY PARAMETERS DURING A DIFFERENT INTENSITY OF PHYSICAL EXERCISE IN THE POPULATION OF STUDENTS
}

\begin{abstract}
:
Introduction: It is known that music can reduce the sense of fatigue and pain during physical exercise as well as to affect the motivation. Physiological effects of music during submaximal intensity cardiopulmonary exercise tests can be examined using a treadmill.
\end{abstract}

Aim: In this study we examined the impact of asynchronous music on cardiopulmonary parameters of students, while they were running on the treadmill on the moderate to high load. Also we examined the difference between these two conditions.

Materials and methods: This study involved 6 students from the Faculty of Medicine in Belgrade, which were tested in the conditions with and without music. In order to determine maximal oxygen consumption, maximal exercise tests were conducted in advance. During the testing phase, cardiopulmonary parameters were measured in two different conditions: on $70 \%$ of maximal measured $\mathrm{VO}_{2}$ values and on $90 \%$ of previously measured maximal $\mathrm{VO}_{2}$ values respectively.

Results: The breathing frequency, ventilation, exhaled $\mathrm{CO}_{2}$ volume, blood pressure were significantly increased $(\mathrm{p}<0.05)$ in terms of music on a moderate rate of $70 \% \mathrm{VO}_{2}$ max. On high rate of $90 \% \mathrm{VO}_{2}$ max , there has been no significant changes to the parameters $(\mathrm{p}>0.05)$ except a decrease in blood pressure and minimal increase in $\mathrm{VO}_{2} / \mathrm{kg}$.

Conclusion: This study confirmed the influence of music on cardiopulmonary parameters and demonstrated that it can affect respiratory variables only on moderate loads. Results showed that music does not affect respiratory variables on high loads, but there was a significant decrease in blood pressure in both, moderate and high loads.

Key words: music, sport, breathing, ventilation, $\mathrm{VO}_{2}, \mathrm{VCO}_{2}$, blood pressure

1 Clinical Centre of Serbia, Doktora Subotića, Belgrade. 


\section{Introduction}

It is known that music can reduce the feeling of fatigue and pain during physical exercise (1) and influence the motivation in the population of athletes (2-4). Music achieves its ergogenic effects in several ways. It can affect thoughts by decreasing feeling of exhaustion through motivation, directing attention away from unpleasant stimuli such as pain and peripheral fatigue $(5,6)$. Also, music over the limbic nervous system can act as stimulant or sedative to motor and autonomous nerve centers in the cortex and brain stem by regulating their functions $(7,8)$. Finally, we should mention the synchronization of the movement and rhythm of the music during the exercise as one of the factors that may have beneficial psychological effects (4). However, in studies on the influence of music on exercise results, it has been proven that music can have both a positive and negative impact on the functional and psychological parameters, which depends on the selection of used songs. When we discuss about music and exercise, it is important to reflect the cumulative impact of the factors that affect the results: type and form of exercises, music characteristics, synchronization of steps and rhythms. Some of the scientific studies found that music has no effect in high load exercise (higher than $80 \%$ maximum functional abilities, i.e. maximum oxygen consumption during exercise, $\mathrm{VO}_{2} \max$ ), while in moderate and lower loads it has positive results influenced by the adequate selection of music (9). The results of these studies emphasize the selection of music. Based on the preceding results, we can conclude that the results during the exercises are influenced by the load and used protocol during testing phase and the characteristics of music such as tempo and harmony $(6,11)$. Given the lack of literature that directly compares the influence of music to moderate and high loads, in this study we wanted to examine the impact of the music on a change of cardiopulmonal parameters during cardiopulmonary exercise test (CPET) on a treadmill under moderate and high loads. Also, we examined the difference between testing conditions, with music and without music.

\section{Materials and methods}

\section{Subjects}

The research involved 6 students from the Faculty of Medicine in Belgrade aged from 20 to 22 years. All participants were thoroughly aware of the procedures and objectives of the research and their signature gave the consent for testing. Before the survey, each participant filled out a document with personal data, medical and athletic anamnesis 


\section{Study design}

Before the CPET, all participants made personal playlist of 5 songs that they considered motivational during exercise. Test was performed in three phases, i.e. each participant had performed 3 tests separated by a daily break. The first phase of the study was to determine maximum oxygen consumption $\left(\mathrm{VO}_{2} \mathrm{max}\right)$ during the maximum, progressive, continuous test on the treadmill. The test was preceded by anthropological measurements, ECG, blood pressure measurements (TA) and physical examination. After determining $\mathrm{VO}_{2}$ max for each person, we calculated the speed of the treadmill in which the oxygen consumption of $70 \%$ and $90 \%$ of $\mathrm{VO}_{2}$ max was reached. This speed was a referent load for performing a second phase of research, or a submaximal test. After a day of pause, our subjects were tested again, but this time on moderate and high submaximal loads. Participants were in a random choice divided into two groups: a group running at a moderate load of $70 \% \mathrm{VO}_{2} \max$ and a group that ran at a high load of $90 \% \mathrm{VO}_{2}$ max. The submaximal tests were carried out in each group 2 times, in conditions with the individual selection of stimulating music and in conditions without music. During all tests we measured cardiopulmonal parameters.

\section{Anthropological measurements}

Following anthropometrical parameters were measured for each subject: body weight (BW) in accuracy of $0.1 \mathrm{~kg}$ (Body Composition Analyzer, Seoul, Korea), body height (BH) measured (Seca709, Hamburg, Germany) with the accuracy of 0,1 cm. The percentage of body fat and muscles were evaluated using InBody230 scales.

\section{Cardiopulmonal measurements}

Measured functional parameters were: heart rate (HR - in peace, at maximum load, and after three minute recovery), consumption of oxygen $\left(\mathrm{VO}_{2} / \mathrm{kg}\right)$, respiratory exchange ratio (RER), ventilation (VE), breathing frequency (BF), the volume of the Exhaled $\mathrm{CO}_{2}\left(\mathrm{VCO}_{2}\right)$, blood pressure (TA) which was manually measured (immediately before the test and 3 minutes after the test was ended). The parameters were measured using the portable ergospirometer (The Oxycon Mobile OM, Jäger, Würzburg, Germany).

\section{Maximual Load Test}

Before performing each test, the calibration of the volume sensors was performed, for the sensor for oxygen and carbon dioxide (with a syringe of known volume 
and with the known gas mixture, respectively). All participants performed maximal progressive protocol on the treadmill (HP Cosmos Ltd, Nussdorf, Germany).

The protocols were individually adapted for each participant based on the previous questionnaire, which have provided insight into the physical possibilities of the subjects, so that the expected maximal values could be reached in the interval between 8-12 minutes of testing. Installation of the gas analysis equipment and 3 minutes warm up on treadmill at speed up to $5 \mathrm{~km} / \mathrm{h}$, was followed by the phase of gradual increase in treadmill speed up to the end of the testing phase. The analysis of the gas exchange was carried out using breath by breath method (The Oxycon Mobile OM, Jäger, Würzburg, Germany) with continuous monitoring of the 12 channel ECG. Heart rate was determined through ECG recording. Indicators for the ending of the test were: subjective fatigue of participants, achievement of estimated maximal heart frequency and the value of the respiratory exchange ratio $($ RERmax $) \geq 1.10$.

\section{Submaximal Load Test}

The submaximal load test was performed using a protocol that included 1 minute and 30 seconds of warm up period on a treadmill. After the warm up period, we have increased the speed of treadmill up to the previously calculated values. The referent speed value was the one at which the subject has reached $70 \%$ or $90 \%$ of the previously established $\mathrm{VO}_{2}$ max. After the warm up, subjects ran for the following six minutes on a given load. Three participants ran at a moderate load of $70 \%$ while the second group of three participants ran at a high load of $90 \%$. Six minutes of running were followed by a 3-minute resting phase on the treadmill. The test was repeated in 2 phases that were separated with a minimum of $24 \mathrm{~h}$ break. During the first arrival, participants listened to the music during the testing phase. Personalized playlists made in the telephone application (Deezer, Paris, France) were applied by wireless headphones (Sony WH-CH400, Japan).

\section{Statistical analysis}

All data is displayed using standard descriptive statistics, presented as an arithmetic mean with standard deviation. The differences of arithmetic mean of parameters during testing in the presence and absence of music are presented using $\mathrm{T}$ test for dependent samples and non-parametric test for dependent samples in case of a normal distribution absence. All statistical analyses were made using the Statistic package for social Sciences 15 (SPSS15) program. 


\section{Results}

\section{Anthropometric measurements}

The demographic and anthropometric parameters of participants are presented in the Appendix I.

\section{Cardiopulmonal measurements}

The implementation of statistical tests for two related samples showed that there is a statistically significant difference in the breathing frequency $(\mathrm{BF})$, ventilation (VE), the volume of exhaled $\mathrm{CO}_{2}\left(\mathrm{VCO}_{2}\right)$, blood pressure (TA) at the load of $70 \%$ $\mathrm{VO}_{2} \max (\mathrm{p}<0.05)$. The average breathing frequency in the presence of music was $39.89 \pm 6.04$ breaths per minute, while in the absence of music amounted to $33.07 \pm$ 5.81 breaths per minute (appendix, III). In the presence of music, the average ventilation has reached the value of $77.256 \pm 28.56 \mathrm{~L} / \mathrm{min}$, while in the absence of music it was $68.15 \pm 26.85 \mathrm{~L} / \mathrm{min}$ (appendix, IV). Exhaled $\mathrm{CO}_{2}$ volume in the presence of music was $2303 \pm 959.20 \mathrm{~mL} / \mathrm{min}$, while in the absence of music was $2122.1 \pm$ $955.57 \mathrm{~mL} / \mathrm{min}$ (appendix, V). The average systolic blood pressure in the presence of music was $153.33 \pm 5.33 \mathrm{mmHg}$ and in the absence of music was $166.67 \pm 11.54$ $\mathrm{mmHg}$ (appendix, VIII). The average diastolic blood pressure in the presence of music showed the value of $83.33 \pm 5.33 \mathrm{mmHg}$, and $90 \pm 11 \mathrm{mmHg}$ in the absence of music (appendix IIc, VII). Statistically significant difference for the consumption of oxygen per kilogram and respiratory volume was not found (p. 0.05).

The application of the student $\mathrm{T}$ test for two related samples showed statistically significant difference in oxygen consumption $\left(\mathrm{VO}_{2} / \mathrm{kg}\right)$ and blood pressure at the load of $90 \% \mathrm{VO}_{2} \max (\mathrm{p}<0.05)$. The arithmetic mean of oxygen per kilogram in the presence of music was $34.96 \pm 3.97 \mathrm{~mL} / \mathrm{min} / \mathrm{kg}$, while in the absence of music it was $34.46 \pm 4.87 \mathrm{~mL} / \mathrm{min} / \mathrm{kg}$ (appendix, IIb, VI). The average systolic blood pressure in the presence of music showed value of $163.33 \pm 5.77 \mathrm{mmHg}$, while in the absence of music it was $173.33 \pm 5.77 \mathrm{mmHg}$ (appendix IId, VIII). The average diastolic blood pressure in the presence of music was $84.67 \pm 5.50 \mathrm{mmHg}$, and $90 \pm 0.00 \mathrm{mmHg}$ in the absence of music (appendix IId, VII). The other parameters have not showed a statistically significant difference.

\section{Discussion}

The aim of this study was to examine the influence of music on the functional parameters of the cardiovascular and respiratory system during submaximal CPET 
at different levels of intensity. We posted the hypothesis that stimulating music will lead to changes in gas exchange parameters during a moderate load test. We did not expect this change at a high-load test. The results showed that there is a significant increase in breathing frequencies when running in conditions with music on the load of $70 \% \mathrm{VO}_{2} \max$. Our result is in accordance with the results of the studies of Salimpoor $(12,13)$ and Benedek (14) who have proven that music that causes a positive emotion, increases the respiratory frequency, cardiac frequency and reduces blood pressure. It is considered that music achieves this through the limbic system activity, by causing dopamine release and a consequentially caused positive emotional state is affecting autonomous nerve centers through the connections that these two systems share (13-15). As the breathing frequency is controlled through the signal of an autonomous nervous system originating from the respiratory center at the level of the prolonged spinal cord $(16,17)$ we can assume that the music indirectly had an impact on the increase of the breathing frequency of $20.61 \%$ during the test at $70 \%$ load compared with the same load, but without music. Increased breathing frequencies were followed by increased ventilation of $13.35 \%$ at the load of $70 \%$ $\mathrm{VO}_{2}$ max values. Based on this increase, we can see that the amount of air inhalation per minute corresponds to the increase of the respiratory frequency while listening to stimulating music during running. During the load of $90 \%$ of $\mathrm{VO}_{2} \max$, as we expected, there was no change in these parameters. Despite the numerical increase of ventilation in the results, no statistical difference was obtained in the consumption of oxygen when subjects had listened to stimulating music at $70 \%$ test, while on the test of $90 \%$ VO2max, value had an increase of $1.75 \%$. Oxygen consumption in the same load was examined by Lim (18) and Terry (19) during the test on treadmill and cycling. In the study of Lim (18), there was no difference in the consumption of oxygen on the submaximal strain, while in the Terry (19) study, it was proven. Based on the results of previously mentioned studies and our research we see that there is a disagreement on the influence of music on $\mathrm{VO}_{2}$ consumption, which is likely due to different conditions in which the test was performed, the selection of exercises, music and adaptions of subjects to the test. By comparing each other's changes in the respiratory variables, we can confirm that the impact of stimulating music to increase in the frequency of breathing and ventilation does not have to be in a correlation with the increase of $\mathrm{VO}_{2}$. This indicates that a change in the number of breaths per minute does not affect the $\mathrm{VO}_{2}(20)$. However, the results showed that there was an increase in the amount of the exhaled $\mathrm{CO}_{2}$ during test with the music due to the increasing of ventilation in the same conditions (appendix V). It is evident that the chosen motivational music during the test raises an emotional response that affects the respiratory center by increasing the breathing frequency, ventilation and the amount of exhaled $\mathrm{CO}_{2}$. This was not the case at the load of $90 \%$ $\mathrm{VO}_{2}$ max because the influence of music is dominated by increased physical effort. 
The Tenenbaum (10) study showed that music does not have a significant impact on the autonomous nervous system and the regulation of breathing during the exercise on the load higher than $80 \% \mathrm{VO} 2 \mathrm{max}$ which our scores have confirmed. In cases of $70 \%$ and $90 \%$ of $\mathrm{VO}_{2} \max$, there was a difference of $2.25 \%$ and $2 \%$ in the RER, but it is was not statistically significant and cannot be attributed to the impact of music on the respiratory ratio. The influence of music on hemodynamic parameters was investigated in many studies, both in rest and exercise $(1,21)$. In studies conducted by Noguchi (22) and Loomba (21) it was proven that music can lower the blood pressure through the already mentioned relax effects that it achieves through the connection of the limbic and autonomous nervous system. The results of our studies have confirmed that there is a significant difference both on high and moderate loads in blood pressure, but the difference is more noticeable at lower load. According to our findings, in the available literature, there is no difference in the selection of music and the manner of its effect on the breathing frequency during exercise, but the available results are limited (22) so a future research needs examine the influence of motivational, stimulating and demotivational, sedative music and compare them. Also, in this survey we examined only motivating factor of music with the absence of running and rhythms. Sound wave is made of frequency, strength, color, spread speed, while music as a sum of audio stimuli consists of melody, rhythm, harmony, tempo. Due to the extensive complexity of the music itself and the effect on physiological parameters, it is not simple to explore and explain its influence through one research due to the cumulative effects of the previously mentioned characteristics that must be individually examined and compared.

\section{Conclusion}

This research confirmed the impact of the music on cardiopulmonal parameters. It has showed the change in frequency of breathing, ventilation, the amount of exhaled $\mathrm{CO}_{2}$ the reduction of pressure on moderate load, while the changes of other parameters were not statistically significant. On high loads, there was a minimal increase in oxygen consumption and the reduction of blood pressure, without significant differences in other parameters.

Our plan research is to examine the effect of music on a larger group of subjects. We believe that the expansion of research methodology (including the analysis of EEG, EMG signal, lactates and hormone measurements) will improve the understanding of influence of music on different functional parameters during physical exercise. 


\section{References}

1. Szmedra L, Bacharach DW. Effect of music on perceived exertion, plasma lactate, norepinephrine and cardiovascular hemodynamics during treadmill running. Int J Sports Med. 1998; 19(1): 32-7.

2. Hernandez-Peon R, Brust-Carmona H, Penaloza-Rojas J, Bach-Y-Rita G. The efferent control of afferent signals entering the central nervous system. Ann N Y Acad Sci. 1961; 89: 866-82.

3. Brooks GA, Fahey TD, White TP BK. Exorcise Physiology: Human Bioenergetics and Its Aplications. Exercise physiology. 2000; 58-60.

4. Karageorghis CI, Priest D-L. Music in the exercise domain: a review and synthesis (Part I). Int Rev Sport Exerc Psychol. 2012 Mar; 5(1): 44-66.

5. Yamashita S, Iwai K, Akimoto T, Sugawara J, Kono I. Effects of music during exercise on RPE, heart rate and the autonomic nervous system. J Sports Med Phys Fitness. 2006 Sep; 46(3): 425-30.

6. Karageorghis CI, Priest D-L. Music in the exercise domain: a review and synthesis (Part II). Int Rev Sport Exerc Psychol. 2012 Mar; 5(1): 67-84.

7. Bigliassi M, León-Domínguez U, Buzzachera CF, Barreto-Silva V, Altimari LR. How does music aid $5 \mathrm{~km}$ of running? J strength Cond Res. 2015 Feb; 29(2): 305-14.

8. Bigliassi M, Silva VB, Karageorghis CI, Bird JM, Santos PC, Altimari LR. Brain mechanisms that underlie the effects of motivational audiovisual stimuli on psychophysiological responses during exercise. Physiol Behav. 2016 May 1; 158: 128-36.

9. Pujol TJ, Langenfeld ME. Influence of music on Wingate Anaerobic Test performance. Percept Mot Skills. 1999 Feb; 88(1): 292-6.

10. Tenenbaum G, Lidor R, Lavyan N, Morrow K, Tonnel S, Gershgoren A. Dispositional and task-specific social-cognitive determinants of physical effort perseverance. J Psychol. 2005 Mar; 139(2): 139-57.

11. Szabo A, Small A, Leigh M. The effects of slow- and fast-rhythm classical music on progressive cycling to voluntary physical exhaustion. J Sports Med Phys Fitness. 1999 Sep; 39(3): 220-5.

12. Salimpoor VN, Benovoy M, Longo G, Cooperstock JR, Zatorre RJ. The rewarding aspects of music listening are related to degree of emotional arousal. Lauwereyns J, editor. PLoS One. 2009 Oct 16; 4(10): e7487.

13. Salimpoor VN, Benovoy M, Larcher K, Dagher A, Zatorre RJ. Anatomically distinct dopamine release during anticipation and experience of peak emotion to music. Nat Neurosci. 2011 Feb 9; 14(2): 257-62.

14. Benedek M, Kaernbach C. Physiological correlates and emotional specificity of human piloerection. Biol Psychol. 2011 Mar; 86(3): 320-9.

15. Menon V, Levitin DJ. The rewards of music listening: response and physiological connectivity of the mesolimbic system. Neuroimage. 2005 Oct 15; 28(1): 175-84.

16. Williamson JW. The relevance of central command for the neural cardiovascular control of exercise. Exp Physiol. 2010; 95(11): 1043-8. 
17. Bechbache RR, Duffin J. The entrainment of breathing frequency by exercise rhythm. J. Physiol. 1977 Nov; 272(3): 553-61.

18. Lim HBT, Karageorghis CI, Romer LM, Bishop DT. Psychophysiological effects of synchronous versus asynchronous music during cycling. Med Sci Sports Exerc. 2014 Feb; 46(2): 407-13.

19. Terry PC, Karageorghis CI, Saha AM, D'Auria S. Effects of synchronous music on treadmill running among elite triathletes. J Sci Med Sport. 2012 Jan; 15(1): 52-7.

20. Nalbandian M, Radak Z, Taniguchi J, Masaki T. How Different Respiratory Rate Patterns affect Cardiorespiratory Variables and Performance. Int J Exerc Sci. 10(3): 322-9.

21. Loomba RS, Arora R, Shah PH, Chandrasekar S, Molnar J. Effects of music on systolic blood pressure, diastolic blood pressure, and heart rate: a meta-analysis. Indian Heart J. 2012 May; 64(3): 309-13.

22. Noguchi K, Masaoka Y, Satoh K, Katoh N, Homma I. Effect of Music on Emotions and Respiration. Showa Univ J Med Sci. 2012; 24(1): 69-75. 


\section{Appendix I}

Table 1. Demographic and anthropometric characteristics of subjects

\begin{tabular}{|c|c|c|c|c|c|c|c|}
\hline Subject & Sex & Age & BH (cm) & BW (kg) & $\begin{array}{c}\text { BMI } \\
\left.\mathbf{( k g} / \mathbf{m}^{2}\right)\end{array}$ & $\mathbf{\% B F}$ & \%muscle \\
\hline $\mathbf{1}$ & $\mathrm{f}$ & 21 & 167 & 54.4 & 19.5 & 15.9 & 45.95 \\
\hline $\mathbf{2}$ & $\mathrm{f}$ & 21 & 173 & 61.1 & 20.4 & 21.7 & 43.69 \\
\hline $\mathbf{3}$ & $\mathrm{m}$ & 21 & 180 & 79.6 & 24.6 & 13 & 49.49 \\
\hline $\mathbf{4}$ & $\mathrm{m}$ & 20 & 180 & 78.6 & 24.3 & 13.4 & 49.9 \\
\hline $\mathbf{5}$ & $\mathrm{m}$ & 21 & 173 & 66.5 & 22.2 & 14 & 48.72 \\
\hline
\end{tabular}

$\mathrm{BH}$ - Body Height; BM - Body Mass; BMI - body mass index; \%BF - percent body fat; sex-f-female; sex-m-male

\section{Appendix IIa}

Table 2. Functional parameters of the subjects at the load of $70 \%$ VO2max values

\begin{tabular}{|c|c|c|c|}
\hline Variables & Music & Without music & $\mathbf{p}$ \\
\hline BF & $39.89 \pm 6.04$ & $33.07 \pm 5.81$ & $<0.05^{*}$ \\
\hline $\mathbf{V E}(\mathbf{L} / \mathbf{m i n})$ & $77.25 \pm 28.55$ & $68.15 \pm 26.85$ & $<0.05^{* *}$ \\
\hline $\mathbf{V O} / \mathbf{k g}(\mathbf{m l} / \mathbf{m i n} / \mathbf{k g})$ & $34.96 \pm 3.97$ & $34.46 \pm 4.87$ & $>0.05$ \\
\hline $\mathbf{V C O}(\mathbf{m l} / \mathbf{m i n})$ & $2304.43 \pm 798.5$ & $2122.1 \pm 797.45$ & $<0.05^{* *}$ \\
\hline $\mathbf{R E R}$ & $0.88 \pm 0.06$ & $0.89 \pm 0.06$ & $>0.05$ \\
\hline
\end{tabular}

Variables are displayed as $\mathrm{X} \pm \mathrm{SD}$; BF (Breathing Frequency) - respiratory frequency; VE (Ventilation) - ventilation; VO2/kg-oxygen consumption per unit of body mass; VCO2 - volume of exhaled CO2; RER (Respiratory Exchange Ratio)

Student $\mathrm{T}$ test $* \mathrm{p}<0,05$

Wilcox test 


\section{Appendix IIb}

Table 3. Functional parameters of respondents in the load of $90 \% \mathrm{VO}_{2}$ max values

\begin{tabular}{|c|c|c|c|}
\hline Variables & Music & Without music & $\mathbf{p}$ \\
\hline $\mathbf{B F}$ & $47.48 \pm 5.80$ & $47.10 \pm 7.17$ & $>0.05$ \\
\hline $\mathbf{V E}(\mathbf{L} / \mathbf{m i n})$ & $112.71 \pm 19.23$ & $108.05 \pm 22.94$ & $>0.05$ \\
\hline $\mathbf{V O}_{2} / \mathbf{k g}(\mathbf{m l} / \mathbf{m i n} / \mathbf{k g})$ & $43.15 \pm 2.71$ & $42.41 \pm 2.78$ & $<0.05^{*}$ \\
\hline $\mathbf{V C O} \mathbf{( m l} / \mathbf{m i n})$ & $3117.33 \pm 259.04$ & $2999.43 \pm 348.62$ & $>0.05$ \\
\hline RER & $0.99 \pm 0.02$ & $1.01 \pm 0.04$ & $>0.05$ \\
\hline
\end{tabular}

Variables are displayed as $\mathrm{X} \pm \mathrm{SD}$; $\mathrm{BF}$ (Breathing Frequency)-respiratory frequency; $\mathrm{VE}$ (Ventilation); $\mathrm{VO}_{2} / \mathrm{kg}$ - oxygen consumption per $\mathrm{kg} ; \mathrm{VCO}_{2}-$ volume of exhaled $\mathrm{CO}_{2}$; RER (Respiratory Exchange Ratio)

Student $\mathrm{T}$ test $* \mathrm{p}<0,05$

Wilcox test $* * \mathrm{p}<0,05$

\section{Appendix IIc}

Table 4. The value of blood pressure of respondents in the load of $70 \% \mathrm{VO}_{2}$ max values

\begin{tabular}{|c|c|c|c|}
\hline Variables & Music & Without music & p \\
\hline SP(mmHg) & $153.33 \pm 5.77$ & $166.67 \pm 11.54$ & $<0.05^{*}$ \\
\hline DP(mmHg) & $83.33 \pm 5.77$ & $90.00 \pm 0.00$ & $<0.05^{*}$ \\
\hline
\end{tabular}

Variables are displayed as $\mathrm{X} \pm \mathrm{SD}$;

SP-systolic pressure; DP-Diastolic pressure

Student T test $* \mathrm{p}<0,05$

\section{Appendix IId}

Table 5. The value of blood pressure of respondents in the load of $90 \% \mathrm{VO}_{2}$ max values

\begin{tabular}{|c|c|c|c|}
\hline Variables & Music & Without music & p \\
\hline SP(mmHg) & $163.33 \pm 5.77$ & $173.33 \pm 5.77$ & $<0.05^{*}$ \\
\hline DP(mmHg) & $84.67 \pm 5.50$ & $90.00 \pm 0.00$ & $<0.05^{*}$ \\
\hline
\end{tabular}

Variables are displayed as $\mathrm{X} \pm \mathrm{SD}$;

SP-systolic pressure; DP-Diastolic pressure

Student $\mathrm{T}$ test $* \mathrm{p}<0,05$ 


\section{Appendix III}

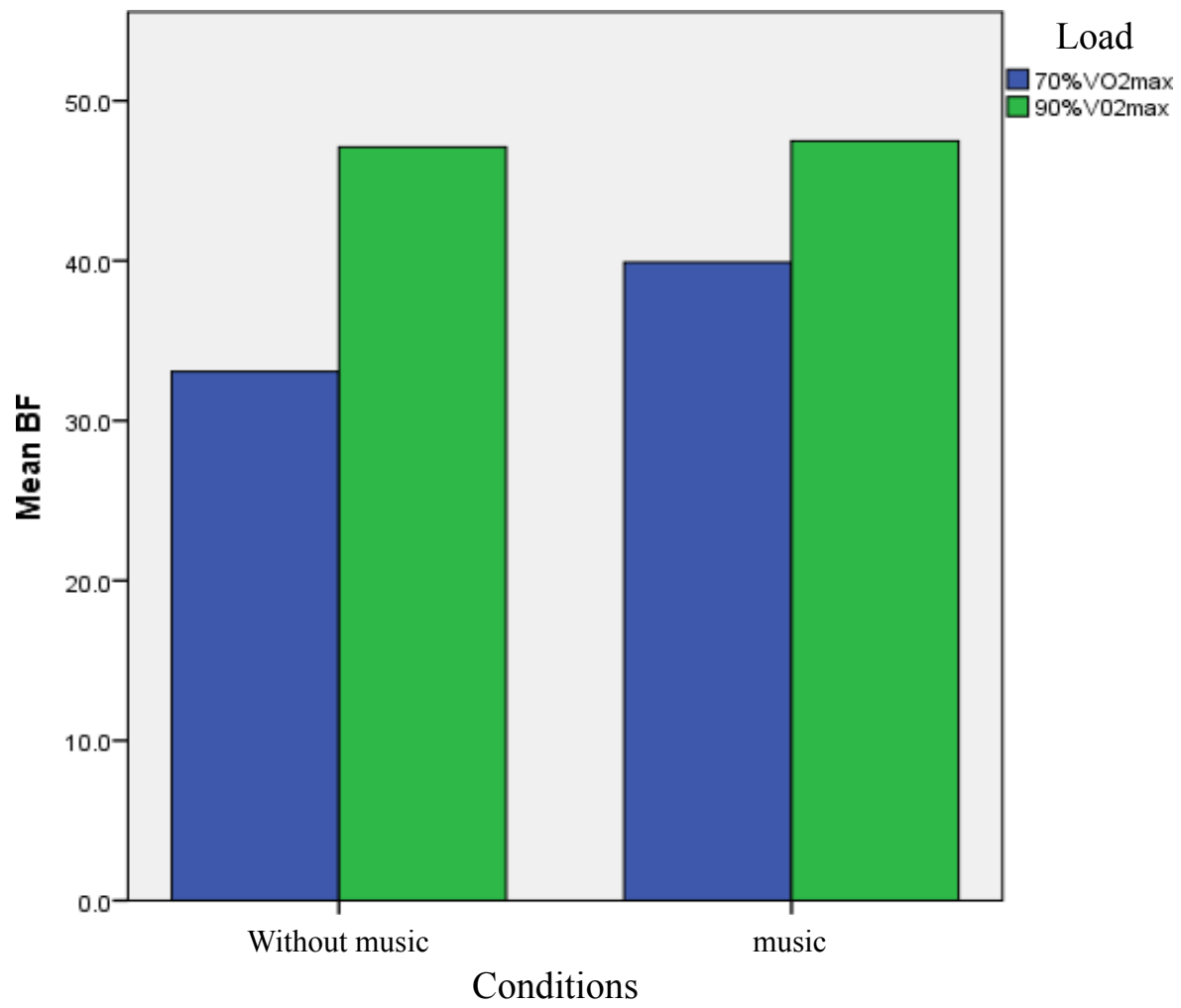

Figure 1. Breathing frequency (BF) in conditions with music and no music at different loads 


\section{Appendix IV}

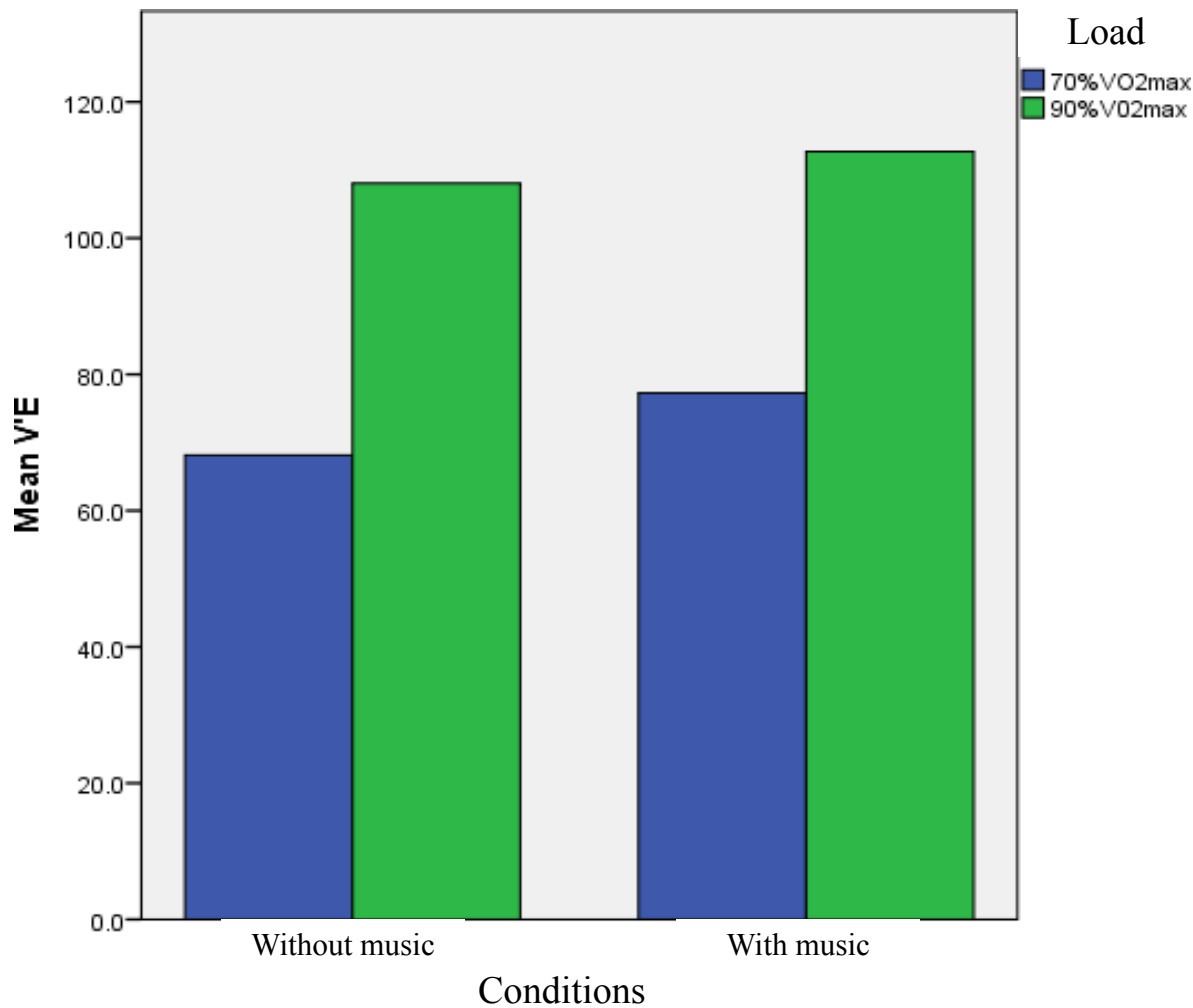

Figure 2. Ventilation (v'e) in conditions with music and no music in loads of $70 \%$ and $90 \%$ $\mathrm{VO}_{2} \max$ values 


\section{Appendix V}

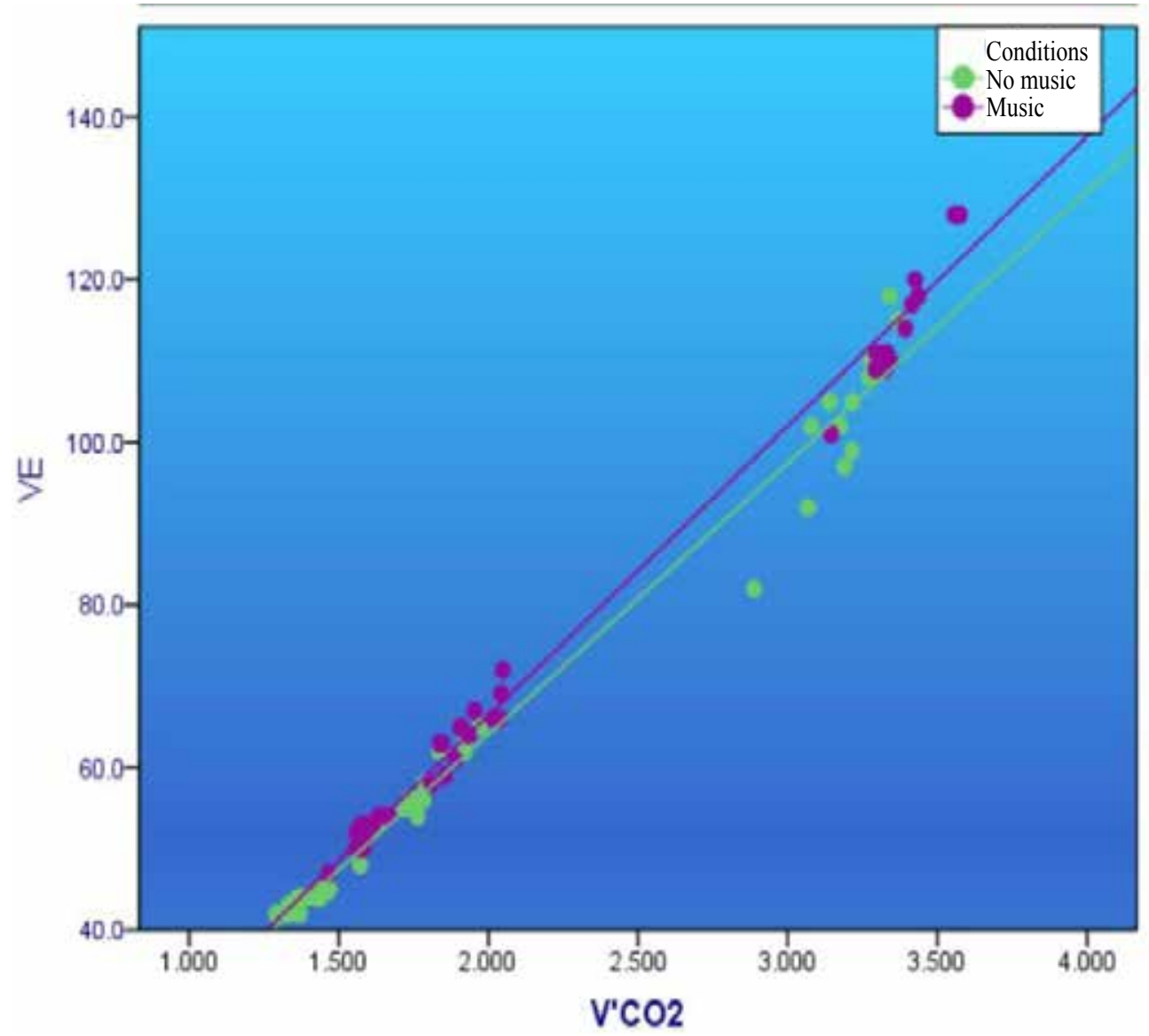

Figure 3. Ratio of exterminated $\mathrm{CO} 2(\mathrm{~V}$ ' $\mathrm{CO} 2)$ and inhaled air in conditions with and without music in the load of $70 \% \mathrm{VO}_{2}$ max values 


\section{Appendix V}

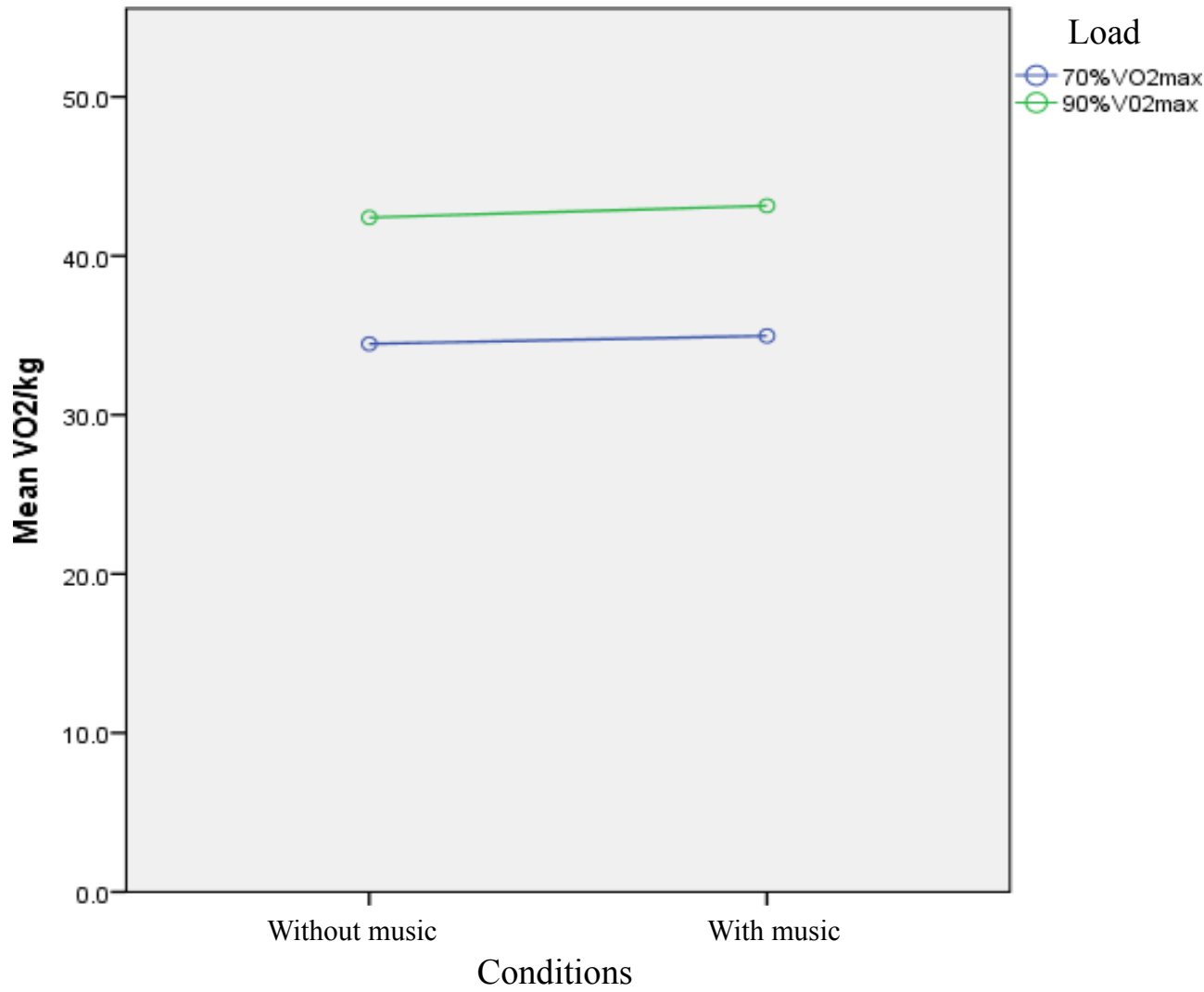

Figure 4. Average consumption of oxygen per $\mathrm{kg}$ of body mass $(\mathrm{VO} 2 / \mathrm{kg})$ in conditions with music and without music at loads of $70 \%$ and $90 \mathrm{VO}_{2}$ max values 


\section{Appendix VII}

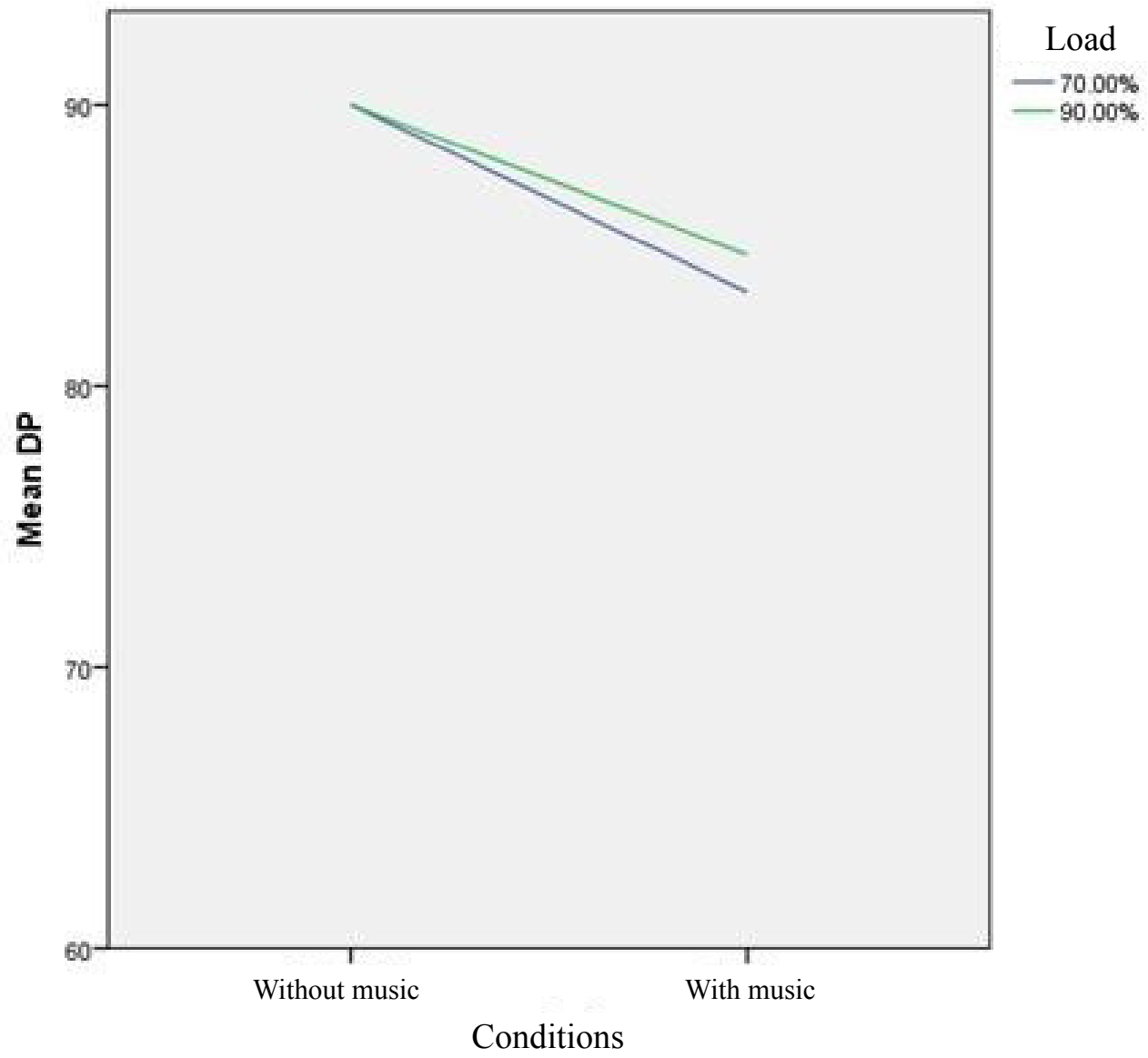

Figure 5. Change of diastolic pressure (DP) in conditions with music and without music in the load of $70 \%$ and $90 \% \mathrm{VO}_{2}$ max values 


\section{Appendix VIII}

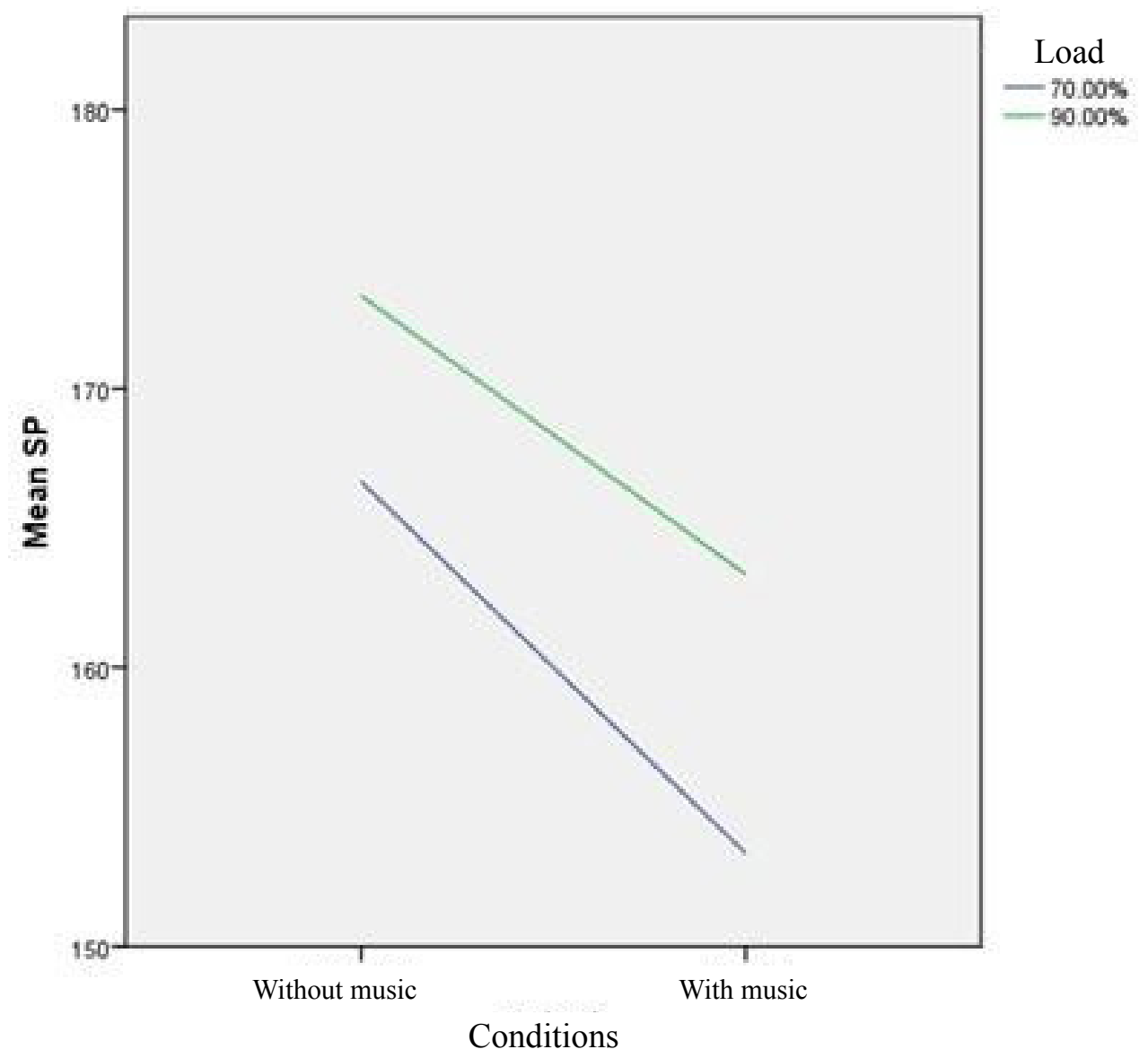

Figure 6. Change of System pressure (SP) in conditions with music and without music at loads of $70 \%$ and $90 \% \mathrm{VO}_{2}$ max values 